\title{
Cinta trans-obturadora (TOT) en la corrección de la incontinencia de orina de esfuerzo. Experiencia de tres años con 200 pacientes
}

\author{
Pardo Schanz $\mathrm{J}^{1,2}$, Ricci Arriola $\mathrm{P}^{2}$, Tacla Fernández $\mathrm{X}^{1}$, Betancourt Ortiz E ${ }^{3}$. \\ ${ }^{1}$ Unidad de Ginecología, Departamento de Obstetricia y Ginecología, Hospital Barros Luco-Trudeau. \\ ${ }^{2}$ Unidad de Uroginecología y Cirugía Vaginal, Departamento de Ginecología, Clínica Las Condes. \\ ${ }^{3}$ Alumno de Postgrado en Obstetricia y Ginecología, Universidad de Chile, Hospital Barros Luco-Trudeau.
}

Actas Urol Esp. 2007;31(10):1141-1147

\section{RESUMEN}

\section{CINTA TRANS-OBTURADORA (TOT) EN LA CORRECCIÓN DE LA INCONTINENCIA DE ORINA DE ESFUERZO. EXPERIENCIA DE TRES AÑOS CON 200 PACIENTES}

Objetivo: Evaluar la seguridad y eficacia del TOT en el tratamiento de la incontinencia de orina de esfuerzo femenina, de acuerdo a nuestra experiencia.

Paciente y Método: Estudio prospectivo de 200 pacientes a los que se realizó TOT en la Unidad de Ginecología del Departamento de Obstetricia y Ginecología del Hospital Barros Luco-Trudeau, entre enero del 2003 y octubre del 2006. Se utilizó malla de polipropileno, macroporo, monofilamento (Prolene). Mediana de edad 52 años, paridad 3.

Resultados: El tiempo operatorio del TOT fue de 15 minutos (mediana). De las 200 pacientes, al mes se constató curación de la incontinencia de esfuerzo en 184 (92\%), 6 (3\%) casos mejoría, 10 (5\%) casos falla de la cirugía. Se presentaron 3 complicaciones intraoperatorias, correspondiendo a lesión de vejiga. Una con la tijera durante la disección del espacio vesico-vaginal hacia el agujero obturador y dos con la aguja. Durante el postperatorio inmediato se evidenció un caso de obstrucción parcial de uretra por inflamación, dos casos de infección urinaria y tres casos con dolor de extremidades inferiores. Durante el postoperatorio tardío en una paciente con cura, como consecuencia de una caída violenta, se reinicia la incontinencia.

Conclusión: De acuerdo a nuestra experiencia con 200 casos, el TOT se presenta como una técnica segura y eficaz en el tratamiento quirúrgico de la incontinencia de orina de esfuerzo.

Palabras clave: TOT. Cinta Trans-obturadora. Cinta suburetral. Incontinencia de orina de esfuerzo. Incontinencia de orina.

\begin{abstract}
TRANS OBTURATOR TAPE (TOT) FOR FEMALE STRESS INCONTINENCE. EXPERIENCE WITH THREE YEARS FOLLOW-UP IN 200 PATIENTS

Objective: To evaluate the security and effectiveness of the TOT in the female incontinence surgical treatment according to our experience.

Patient and Method: Prospective study of 200 patients underwent TOT at Gynecology Unit of the Obstetrics and Gynecology Department in Barros Luco-Trudeau Hospital between January 2003 and October 2006. Polypropilene, macropore, mofilament mesh (Prolene) was used. The mean age was 52 years old, parity 3.

Results: The mean surgical time of TOT was 15 minutes. In 184 (92\%) patients obtained cure, 6 (3\%) improvement and $10(5 \%)$ fails. Three cases of bladder injury occurred (one perforation with the scissor and two with TOT needle during the learning phase). During the immediately postoperative time: one case of partial urethral obstruction, two cases of urinary infection and three with pain of the legs. During the delayed postoperative time a case of fail was observed after trauma.

Conclusion: According to our experience with 200 cases, the TOT appears as a safe and effective technique in the surgical treatment of the stress urinary incontinence.
\end{abstract}


$\mathrm{L}^{\mathrm{a}}$ a primera técnica de cinta suburetral como tratamiento para la incontinencia urinaria de esfuerzo, fue el TVT. Esta cirugía fue descrita por Ulmstein en $1996^{1}$. Se trata de una técnica que requiere de la realización de una cistoscopia de revisión intraoperatoria, para asegurar la indemnidad de la vejiga. Es una cirugía que no está libre de complicaciones, como la perforación vesical ${ }^{2-4}$, de vasos sanguíneos ${ }^{5}$ y de intestino ${ }^{6}$. Durante el 2001 Delorme describe una nueva técnica, el TOT (transobturator-tape). De mayor simpleza y que no requiere la cistoscopia de revisión ${ }^{7}$. Al comparar la eficiencia en la corrección de la incontinencia de orina de esfuerzo, el TOT parece ser igualmente efectivo que el TVT $^{8-10}$

Con el objetivo de evaluar la seguridad y eficacia del TOT en el tratamiento de la incontinencia de orina de esfuerzo, analizamos nuestra experiencia en 200 casos consecutivos.

\section{PACIENTE Y MÉTODO}

Se realizó un seguimiento prospectivo de 200 pacientes a los que se realizó TOT como tratamiento quirúrgico de la incontinencia de orina de esfuerzo, en el Departamento de Ginecología del Servicio de Obstetricia y Ginecología, del Hospital Barros Luco-Trudeau, entre enero del 2003 y octubre del 2006.

Criterio de inclusión: Todas las pacientes ingresadas al estudio debían presentar incontinencia de orina de esfuerzo al menos por un año y de carácter permanente.

Criterio de curación, mejoría y fracaso: El resultado de la cirugía se clasificó según el número de episodios de incontinencia de orina registrados durante el período de observación. Se consideró cura a la ausencia de incontinencia. Mejoría a la presencia de episodios de incontinencia menos que una vez cada dos semanas. Falla a la presencia de episodios de incontinencia más de una vez por semana ${ }^{11}$.

Periodo de observación o seguimiento: hasta octubre del 2006.

Técnica quirúrgica específica: Se utilizó la técnica de TOT descrita originalmente por Delorme ${ }^{7}$. La cinta suburetral utilizada fue de polipropileno, monofilamento, macroporo (Prolene), con un ancho de $10 \mathrm{~mm}$ y $30 \mathrm{~cm}$ de largo. Para su introducción se usaron dos agujas helicoidales, una para cada lado (Fig. 1).

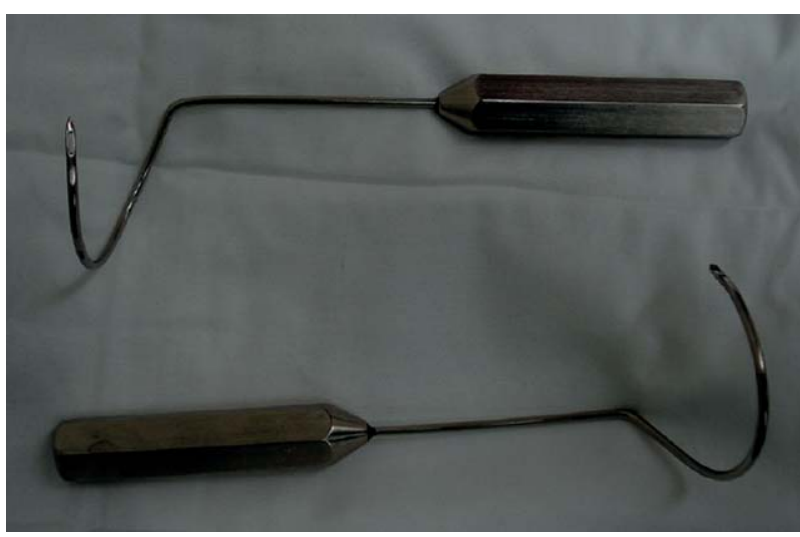

FIGURA 1. Agujas de TOT.

Se utilizó anestesia raquídea para realizar el procedimiento quirúrgico, y se situó a la paciente en la camilla operatoria en posición ginecológica. En dos casos en que se realizó laparoscopía concomitante, se utilizó anestesia general.

Se realizó aseptización de la zona operatoria con povidona yodada. Se colocó una sonda Foley para vaciar la vejiga, la que se dejó hasta el período postoperatorio.

Paso 1: Se marcan los puntos de salida de las agujas. Para ello se traza línea desde el clítoris hasta interceptar el pliegue inguinal.

Paso 2: Se abre la mucosa de la pared vaginal anterior a un centímetro bajo el meato urinario. La incisión es de un centímetro. Se diseca lateralmente y a ambos lados de la zona media uretral con tijera fina, con un ángulo de 45 grados a la línea media, orientándose inmediatamente por debajo de la mucosa, y en dirección a los puntos de salida descritos en el paso 1 (Fig. 2).

Paso 3: Se realiza incisión de $5 \mathrm{~mm}$ en piel de zona inguinal previamente marcada.

Paso 4: Se introduce la aguja helicoidal en la incisión descrita en el punto anterior, dirigiéndola con un dedo localizado en la incisión vaginal. La aguja traspasa la membrana obturadora. Se extrae el extremo de la aguja a través de la incisión vaginal (Fig. 3).

Paso 5: Se pasa el extremo de la cinta por el ojal de la aguja. Se extrae la aguja por la incisión inguinal, realizando el paso inverso a lo descrito en el punto anterior (Fig. 4). Se repite el punto 2 al 5 en el lado contraleteral.

Paso 6: Se ajusta la tensión de la malla. Para lo cual se introduce una tijera entre la uretra y la 


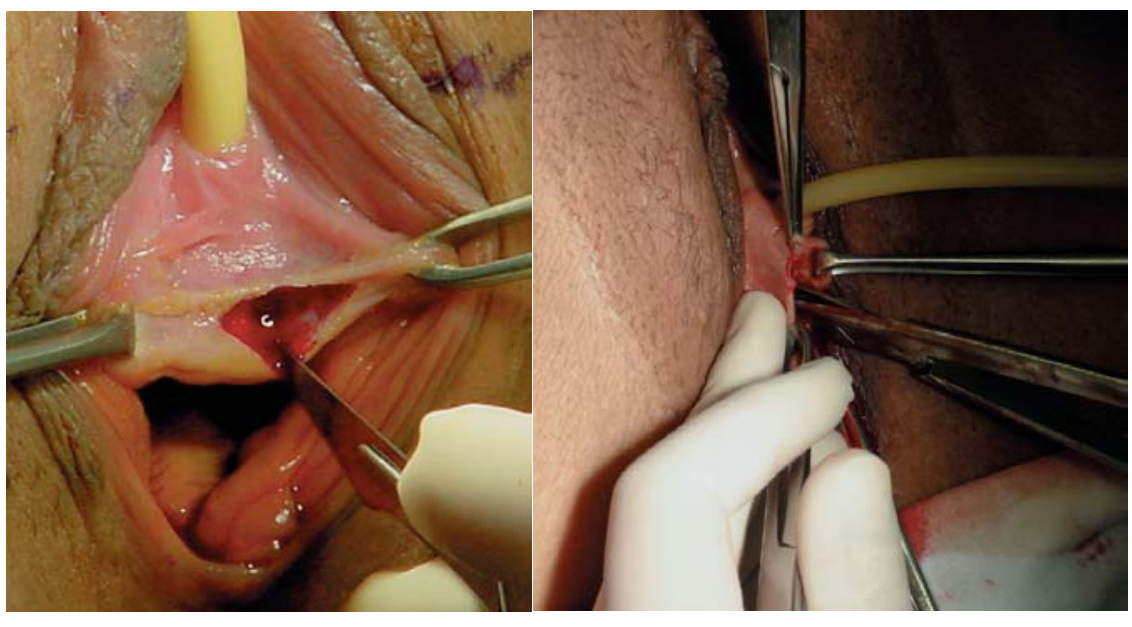

FIGURA 2
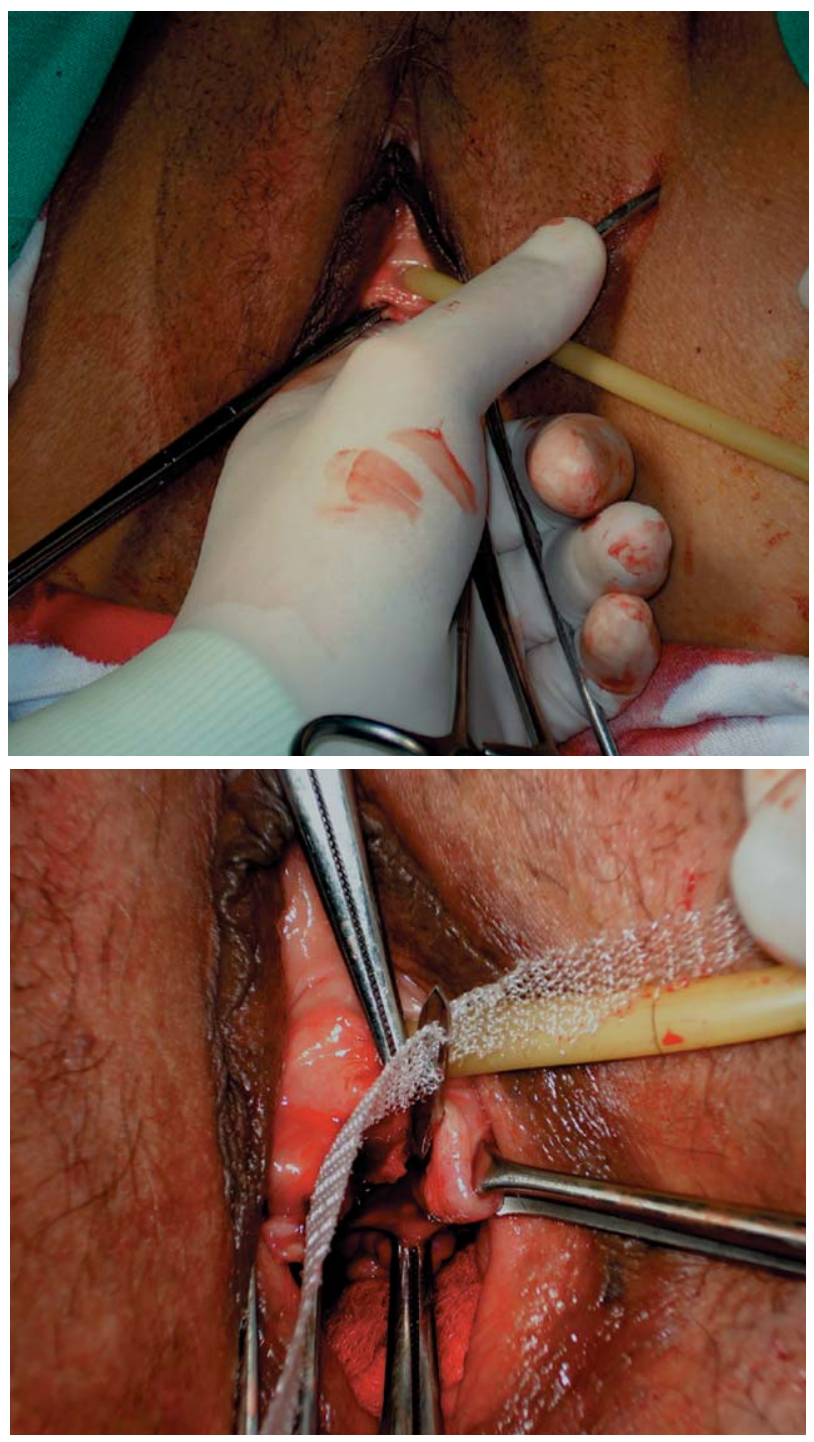

FIGURA 3 malla (Fig. 5). Luego se corta la cinta a nivel subcutáneo, y se sutura con un solo punto en la piel. Finalmente se sutura la mucosa vaginal.

En los casos en que asoció otra cirugía ginecológica, se realizó en un tiempo posterior al TOT.

A todas las pacientes se les explicó previamente, el diagnóstico de su patología, en qué consistía el TOT y en los casos correspondientes, el procedimiento quirúrgico concomitante a realizar. Todas firmaron un consentimiento informado.

Se utilizó cefazolina 1 gramo endovenoso intraoperatorio como profilaxis. La analgesia postoperatoria fue intramuscular el primer día y vía oral a partir del segundo día, con refuerzo intramuscular en caso necesario.

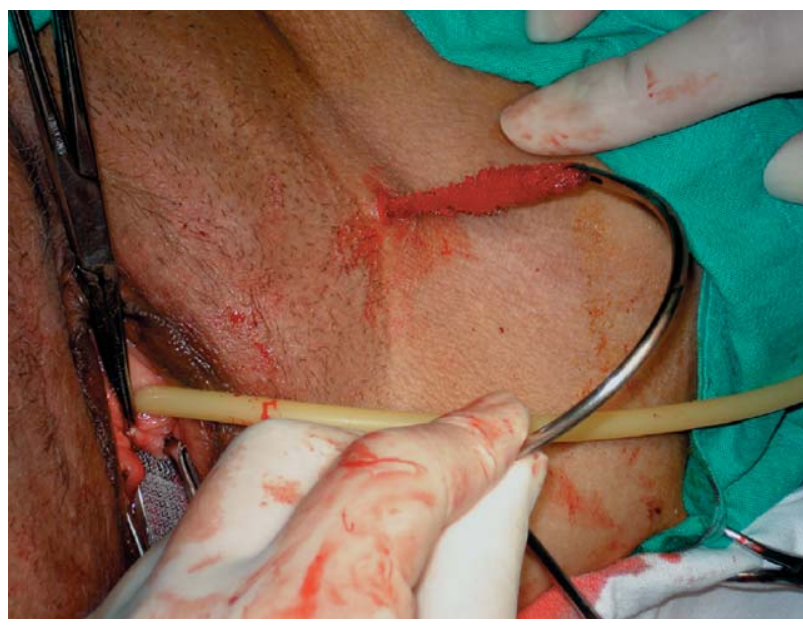

FIGURA 4

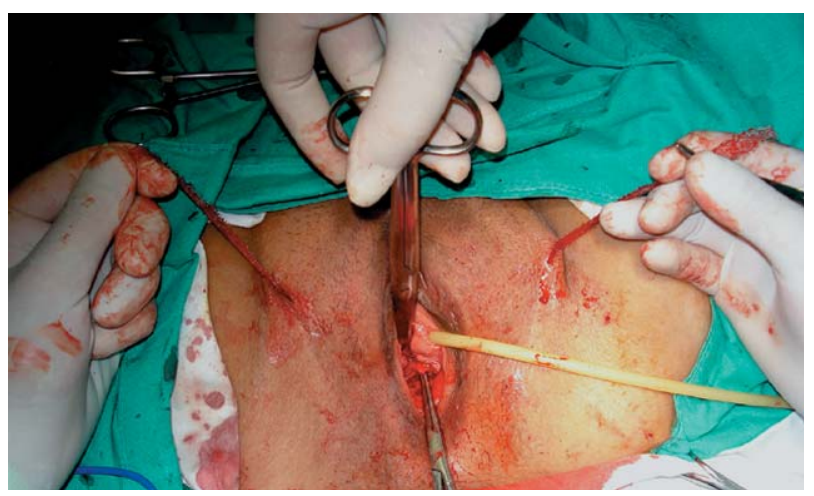

FIGURA 5 
En los casos de TOT sin otra cirugía asociada, se retiró la sonda Foley inmediatamente en el postoperatorio. En los que se asoció histerectomía vaginal y/o colpoperineoplastía anterior y/o posterior, se retiró 12 horas después.

Se dio alta hospitalaria al tercer o cuarto día dependiendo de la cirugía asociada.

Se controló en consulta ambulatoria entre los 7 y 14 días, al mes, a los 3 y 6 meses. Luego en forma telefónica, indicando control ambulatorio en caso de complicación o alteración de la continencia alcanzada.

Características del grupo en estudio: La mediana de edad fue de 52 años, con rango entre 34 y 78 años. Paridad mediana 3 , rango 1 a 8 . Mediana de presentación de la incontinencia de orina de esfuerzo, 24 meses (Tabla 1).

Tabla 1. Características del grupo ingresado al estudio.

\begin{tabular}{ll}
\hline $\begin{array}{l}\text { Número de pacientes ingresados } \\
\text { al estudio }\end{array}$ & 200 \\
Edad & \\
$\quad \begin{array}{l}\text { Rango } \\
\quad \text { Mediana }\end{array}$ & 52 a 78 años \\
Paridad & \\
$\quad$ Rango & 1 a 8 partos \\
$\quad$ Mediana & 3 partos \\
Presentación de IOE (mediana) & 24 meses \\
Periodo de observación & enero 2003 a \\
& octubre 2006 \\
\hline
\end{tabular}

\section{RESULTADOS}

Se indicó el TOT en 120 casos por incontinencia de orina pura, y en 80 casos por incontinencia de orina mixta con predominio de la de esfuerzo. La media de seguimiento para el grupo en estudio fue de 13 meses.

En 98 (49\%) casos se asoció alguna cirugía ginecológica al TOT. La asociación más frecuente fue TOT a colpoperineoplastía posterior, seguida de colpoperineoplastía anterior más posterior (Tabla 2).

El tiempo operatorio del TOT fue de 15 minutos (mediana), con rango entre 7 y 24 minutos.

Complicaciones intraoperatorias: Se presentaron 3 casos, que correspondieron a lesión de vejiga. Una con la tijera al disecar el espacio vesicovaginal hacia el agujero obturador y dos con la
Tabla 2. Cirugía ginecológica asociada a TOT.

\begin{tabular}{lcc}
\hline Cirugia ginecológica asociada a TOT & Casos & $\%$ \\
\hline TOT sólo & 102 & 51 \\
TOT + Colpoperineoplastía anterior & 6 & 3 \\
TOT + Colpoperineoplastía posterior & 26 & 13 \\
$\begin{array}{l}\text { TOT + Colpoperineoplastía anterior y } \\
\text { posterior }\end{array}$ & 24 & 12 \\
TOT + Histerectomia vaginal & 11 & 5,5 \\
TOT + Histerectomia vaginal + & 17 & 8,5 \\
Colpoperineoplastia ant y posterior & 4 & 2 \\
TOT + Histerectomia Abdominal & & \\
TOT + Esterilización Tubaria Pomeroy & 7 & 3,5 \\
Modificada & & \\
TOT + Quistectomía ovárica por & 2 & 1 \\
Laparoscopia & 1 & 0,5 \\
TOT + Colpoperineocleisis & 200 & 100 \\
Total & &
\end{tabular}

aguja de TOT (estas últimas ocurrieron durante la fase de aprendizaje de dos médicos a los que se enseñaba la técnica). No fueron mayores a 2 centímetros y se suturaron en un plano con vycril 30 por vía vaginal. Se dejó Sonda Foley por 10 días con recuperación completa, y sin presentar nueva complicación posterior (Tabla 3).

Complicaciones del postoperatorio inmediato (hasta 7 días después de la cirugía): Un caso de obstrucción parcial de la uretra por edema e inflamación, fue evidenciado durante este período, corrigiéndose a las 48 horas, lográndose una continencia normal total. Se utilizaron anti-inflamatorios por vía intramuscular y oral.

Se presentaron dos casos de infección urinaria. Uno correspondió a una infección de tracto urinario bajo, que se trató con antibióticos vía oral. El otro caso presentó una pielonefritis aguda, que se trató con antibióticos endovenosos por tres días y luego vía oral, completando siete días de tratamiento, con buena respuesta.

Sin relación al TOT, se presentó un caso de hematoma de la cúpula vaginal, con drenaje espontáneo, en una paciente en la que se asoció histerectomía vaginal.

Además se presentaron 3 casos con dolor de extremidades inferiores producto de la posición de abducción durante la cirugía (Tabla 3). 
Tabla 3. Complicaciones presentadas en el grupo en estudio.

\section{Complicaciones Intraoperatorias \\ Tipo: 1 lesión de vejiga con tijera \\ 2 perforaciones de vejiga con la aguja de TOT \\ Resolución: sutura y Sonda Foley}

\section{Complicaciones del Postoperatorio Inmediato}

Tipo: 1 obstrucción parcial de uretra.

Tipo: 2 infecciones urinarias.

Tipo: 3 casos dolor extremidades inferiores

Complicaciones del Postoperatorio tardio

Tipo: Desplazamiento de cinta al 3er mes
Total: 3 casos

\section{Total: 6 casos}

Resolución: Anti-inflamatorios.

Resolución: Antibioticoterapia.

Resolución: Analgésicos.

Total: 1 caso

Resolución: Nueva cirugía.

Complicaciones del postoperatorio inmediato consideradas hasta los siete dias después de la intervención. Complicaciones del Postoperatorio tardio consideradas después de los siete días desde la intervención.

Complicaciones del postoperatorio tardio (después de 7 días de la cirugía): En una paciente con cura de su IOE, al tercer mes de operada sufre una violenta caída en la vía pública. Reiniciando inmediatamente después de este evento la sintomatologia de incontinencia de esfuerzo, que se había logrado curar por completo con la cirugía. Se informó como falla. Al examinarla se sospechó desplazamiento de la cinta (Tabla 3).

Solución de la incontinencia de orina: De las 200 pacientes, al mes se constató curación de la incontinencia de esfuerzo en 184 (92\%) de ellas. En 6 (3\%) casos se presentó mejoría, quedando con incontinencia sólo a grandes esfuerzos. En 10 (5\%) casos se presentó falla de la cirugía, manteniéndose la condición de incontinencia de esfuerzo. Destaca entre los casos con falla, la paciente que pierde la continencia recuperada con el TOT, después de una caída a los tres meses de la cirugía.

Durante los controles ambulatorios después del primer mes (tres y seis meses) no hubo diferencia en la condición de su continencia alcanzada con el TOT inicialmente. Excepto por el caso de trauma que perdió su condición de cura (considerado dentro de los casos con falla) (Tabla 4).

\section{DISCUSIÓN}

La primera publicación con los resultados y seguimientos de los primeros 49 pacientes a los que realizamos esta técnica, fue con un período de observación entre noviembre del 2003 hasta enero del 2005. En esa ocasión se presentó un $96 \%$ de cura, $1 \%$ de mejoría y $2 \%$ de falla ${ }^{12}$. En el presente informe, con un grupo de 200 pa-
Tabla 4. Resultados del TOT sobre la IOE.

Total: 200 pacientes. Seguimiento hasta octubre 2006. Media de seguimiento: 13 meses

\begin{tabular}{lcc}
\hline & Total & Porcentaje (\%) \\
\hline Cura* $^{* *}$ & 184 & 92 \\
Mejoria** & 6 & 3 \\
Falla*** & 10 & 5 \\
\hline
\end{tabular}

*Cura: ausencia de incontinencia

**Mejoría: episodios de incontinencia < que 1 vez cada 2 semanas ***Falla: episodios de incontinencia $>$ que 1 vez por semana Un caso de falla corresponde a paciente con cura de IOE que al tercer mes de postoperatorio, como resultado de traumatismo pierde su continencia lograda después del TOT (cura).

cientes, y con un período de observación hasta noviembre del 2006, se presenta un $92 \%$ de cura, $6 \%$ de mejoría y $5 \%$ de falla. En resumen entre cura y mejoría se presentó un $97 \%$ en el primer informe y un $98 \%$ en el presente, es decir la variación es muy poca, manteniéndose los porcentajes favorables.

Grise et al. ${ }^{13}$ durante este año mostraron una experiencia con 206 pacientes, en las que se logró un $79,1 \%$ de cura, $13 \%$ de mejoría y $7 \%$ de falla.

Los buenos resultados del TOT se han comparado con los de su antecesor, el TVT. Mellier et al. ${ }^{10}$ compararon un grupo de 94 pacientes con TOT, con otro grupo de 99 con TVT. Un seguimiento del grupo de TOT a 12,8 meses mostró un 95\% de satisfacción, mientras que el grupo con TVT con un seguimiento de 29,5 meses, tuvo un $91 \%$ de satisfacción. Un estudio randomizado realizado por DeTayrac et al. ${ }^{8}$ mostraron con urodinamia, a un año de seguimiento, 83,9\% de curación para TOT y 90\% para TVT. 
Existen varios estudios que muestran la eficacia de esta técnica frente a la $\mathrm{IOE}^{2,7,8,10}$. Nuestro estudio con 200 pacientes también demuestra estos buenos resultados.

El TOT es una técnica cuyas modificaciones frente a TVT, reducen el tiempo de cirugía y sus complicaciones ${ }^{10}$. Diferentes estudios muestran tiempos entre $12^{8}, 14^{14}$ y 21 minutos $^{15}$. Nuestra media de tiempo operatorio fue de 15 minutos. Sin lugar a dudas, un tiempo más reducido que el de TVT, ya que no requiere de revisión cistoscópica.

Por otro lado, se trata de una cirugía que al igual que $\mathrm{TVT}^{16}$, puede ser asociada a otras cirugías ginecológicas. Sin embargo, la alta asociación a otras cirugías del suelo pélvico tales como histerectomía vaginal y corrección de prolapso, hace necesario en nuestra opinión, que esta técnica quirúrgica sea realizada por médicos con experiencia en cirugía vaginal.

Actualmente disponemos también de la técnica de TVT-O ${ }^{17}$, que al igual que TOT, considera el paso de las agujas por el agujero obturador, pero desde "adentro hacia fuera" (TOT es de "afuera hacia adentro").

Esta técnica, que es más sencilla de realizar que el TVT, no está libre de complicaciones, aunque son reducidas en número, tal como lo demuestra nuestra serie. La perforación vesical es una de las complicaciones descritas, que se puede evitar vaciando completamente la vejiga con sonda Foley antes de iniciar el procedimiento. En los casos en que se presentó esta complicación, podemos decir que un caso se debió a un vaciamiento incompleto y los otros dos se produjeron durante la curva de aprendizaje de otros médicos a los cuales se les enseñaba esta técnica (en nuestro hospital se realiza docencia universitaria). En nuestra casuística se presentó en 3 casos de 200 (1,5\%), mientras que otros muestran desde casos aislados ${ }^{18}$ hasta 1 en 120 cirugías $(0,8 \%)^{19}$. Sin embargo, en nuestra experiencia no se registraron perforaciones de uretra que han sido informadas en otros manuscritos. Tampoco se observó erosión de mucosa vaginal ni extrusión de la cinta.

Por otro lado, potencial infección que puede afectar la zona operatoria, y ser potenciada por la cinta, es evitada con un protocolo estricto de antibióticos durante la cirugía, además de una técnica depurada. Recordemos que muchas de las erosiones vaginales y exposición de la cinta, son provocadas por una sub-infección. Otra característica de la malla que puede contribuir a evitar la erosión de la mucosa vaginal es la mayor flexibilidad y menor grosor. El Prolene es poco flexible al compararlo con otras mallas de polipropileno, por lo que juegan un rol de mayor importancia todas las medidas que podemos tomar para evitar esta potencial complicación, que teóricamente podría ocurrir con esta malla.

Lo que no se puede discutir, es el tipo de malla que se debe utilizar para disminuir las complicaciones, deben ser mallas de polipropileno, macroporo, monofilamento. El tamaño del poro mayor a 75 micrones, permitirá el paso de macrófagos, para combatir una potencial infección. Poros pequeños y mallas multifilamentos (ofrecen espacios pequeños entre los filamentos) permitirán el paso de bacterias (menos de 1 micrón) y no de los macrófagos. Por otro lado, el material del cual está confeccionada la malla, no da lo mismo, si queremos evitar las complicaciones ${ }^{20}$. En esta experiencia, hemos utilizado polipropileno macroporo monofilamento, es decir una malla tipo I, según la clasificación de mallas protésicas ${ }^{21}$. Sin embargo se trata de Prolene, que no es una malla diseñada específicamente para vagina, que se utiliza para facilitar el acceso a esta técnica quirúrgica con costos menores a pacientes del sistema público de sanidad.

Interesante resulta mencionar el caso de la paciente que una vez lograda la continencia total con el TOT, al mes como producto de un trauma, se provocó la falla del sistema. Sospechando un desplazamiento de la cinta al examen físico. No registrándose casos similares en la literatura médica.

Si comparamos el ángulo descrito bajo la uretra por la cinta, en TOT es más abierto que el de la cinta de TVT. En el primero la cinta queda describiendo una "V", en cambio en el TVT queda en "U”. Esto ha sido comprobado con ultrasonido ${ }^{22}$.

Un estudio comparativo sobre la mantención de la posición de la cinta bajo la uretra, entre pacientes con TVT y TOT a dos años de seguimiento, no demostró diferencias ${ }^{9}$. 


\section{CONCLUSIONES}

De acuerdo al análisis de nuestra experiencia con 200 casos, el TOT es una técnica segura y eficaz, en la corrección quirúrgica de la incontinencia de orina de esfuerzo.

\section{REFERENCIAS}

1. Ulmsten U, Henriksson L, Johnson P, Varhos G. An ambulatory surgical procedure under local anesthesia for treatment of female urinary incontinence. Int Urogynecol J Pelvic Floor Dysfunct. 1996;7(2):81-85.

2. Dargent D, Bretones S, George P, Mellier G. Insertion of a sub-urethral sling through the obturating membrane for treatment of female urinary incontinence. Gynecol Obstet Fertil. 2002;30(7-8):576-578.

3. Hermieu JF, Messas A, Delmas V, Ravery V, Dumonceau O, Boccon-Gibod L. Bladder injury after trans-obturator tape. Prog Urol. 2003;13(1):115-117.

4. Kuuva N, Nilsson CG. A nationwide analysis of complications associated with the tension-free vaginal tape (TVT) procedure. Acta Obstet Gynecol Scand. 2002;81(1):7277.

5. Zilbert AW, Farrell SA. External iliac artery laceration during tension-free vaginal tape procedure. Int Urogynecol J Pelvic Floor Dysfunct. 2001;12(2):141-143.

6. Peyrat L, Boutin JM, Bruyere F, Haillot O, Fakfak H, Lanson Y. Intestinal perforation as a complication of tension-free vaginal tape procedure for urinary incontinence. Eur Urol. 2001;39(5):603-605.

7. Delorme E. Transobturator urethral suspension: miniinvasive procedure in the treatment of stress urinary incontinence in women. Prog Urol. 2001;11(6):1306-1313.

8. De Tayrac R, Deffieux X, Droupy S, Chauveaud-Lambling A, Calvanèse-Benamour L, Fernandez $\mathrm{H}$, et al. A prospective randomized trial comparing tension-free vaginal tape and transobturator suburethral tape for surgical treatment of stress urinary incontinence. Am J Obstet Gynecol. 2004;190(3):602-608.

9. De Tayrac R, Deffieux X, Resten A, Doumerc S, Jouffroy C, Fernandez H. A transvaginal ultrasound study comparing transobturator tape and tension-free vaginal tape after surgical treatment of female stress urinary incontinence. Int Urogynecol J Pelvic Floor Dysfunct. 2006;17(5):446471.

11. Blaivas JG, Jacobs BZ. Pubovaginal fascial sling for the treatment of complicated stress urinary incontinence. J Urol. 1991;145(6): 1214-1218.

12. Schanz Pardo J, Ricci Arriola P, Solà Dalenz V, Tacla Fernández X. Trans-obturator-tape (TOT) for the surgical repair of stress urinary incontinence: our experience. Arch Esp Urol. 2006;59(3):225-232.
13. Grise P, Droupy S, Saussine C, Ballanger P, Monneins F, Hermieu JF, et al. Transobturator tape sling female stress incontinence with polypropylene tape and outside-in procedure: prospective study with 1 year of minimal follow-up and review of transobturator tape sling. Urology. 2006; 68(4):759-763.

10. Mellier G, Benayed B, Bretones S, Pasquier JC. Suburethral tape via the obturador route: is the TOT a simplification of the TVT?. Int Urogynecol J Pelvic Floor Dysfunct. 2004;15(4):227-232.

14. Costa P, Delmas V. Trans-obturator-tape procedure-"inside out or outside in": current concepts and evidence base. Curr Opin Urol. 2004;14(6):313-315.

15. Corticelli A, Corticelli G, Venuti F, Pedretti L, Dodero D. Preliminary experience with transobturator tape for treatment of urinary incontinence in women. Minerva Ginecol. 2005;57(6):637-640.

16. Solà Dalenz V, Pardo Schanz J, Ricci Arriola P, Guiloff Fische E, Chiang Miranda H. TVT associated with other gynecological operations in the same procedure: results and complications. Arch Esp Urol. 2005;58(10):983-988.

17. Solà Dalenz V, Pardo Schanz J, Ricci Arriola P, Guiloff Fische E, Chiang Miranda H. Minimal invasive surgery in female urinary incontinence: TVT-O. Actas Urol Esp. 2006; 30(1):61-66

18. Minaglia S, Ozel B, Klutke C, Ballard C, Klutke J. Bladder injury during transobturator sling. Urology. 2004;64(2): 376-377.

19. Roumeguère T, Quackels T, Bollens R, de Groote A, Zlotta A, Bossche MV, et al. Trans-obturator vaginal tape (TOT) for female stress incontinence: one year follow-up in 120 patients. Eur Urol. 2005;48(5):805-809.

20. Sola V, Pardo J, Ricci P, Guiloff E. Tension free monofilament macropore polypropylene mesh (Gynemesh PS) in female genital prolapse repair. Int Braz J Urol. 2006;32(4): 410-414.

21. Birch C, Fynes MM. The role of synthetic and biological prothesis in reconstructive pelvic floor surgery. Curr Opin Obtet Gynecol. 2002;14(5):527-535.

22. Cotte B, Dumousset E, Boda C, Mansoor A. Comparison of transobturator tape (TOT) and tension-free vaginal tape (TVT) using perineal ultrasound. Gynecol Obstet Fertil. 2006;34(4):928-303.

Correspondencia autor: Dr. P. Ricci Arriola

Unidad de Uroginecología y Cirugía Vaginal

Hospital Barros Luco-Trudeau.

Avenida José Miguel Carrera 3204, San Miguel,

Santiago de Chile. Tel.: (56-2) 5553331

Email autor: pricci@vtr.net

Información artículo: Original - Incontinencia urinaria de esfuerzo femenina

Trabajo recibido: diciembre 2006

Trabajo aceptado: febrero 2007 